\title{
Interoceptive Interaction: An Embodied Metaphor Inspired Approach to Designing for Meditation
}

Meditation is a mind-body practice with considerable wellbeing benefits which can take different forms. Novices usually start with focused attention meditation that supports regulation of attention towards an inward focus or internal bodily sensations and away from external stimuli or distractors. Most meditation technologies employ metaphorical mappings of meditative states to visual or soundscape representations to support awareness of mind wandering and attention regulation, although the rationale for such mappings is seldom articulated. Moreover, such external modalities also take the focus attention away from the body. We advance the concept of interoceptive interaction and employed the embodied metaphor theory to explore the design of mappings to the interoceptive sense of thermoception. We illustrate this concept with WarmMind, an on-body interface integrating heat actuators for mapping meditation states. We report on an exploratory study with 10 participants comparing our novel thermal metaphors for mapping meditation states with comparable ones, albeit in aural modality, as provided by Muse meditation app. Findings indicate a tension between the highly discoverable soundscape's metaphors which however hinder attention regulation, and the ambiguous thermal metaphors experienced as coming from the body, and supported attention regulation. We discuss the qualities of embodied metaphors underpinning this tension and propose an initial framework to inform the design of metaphorical mappings for meditation technologies.

CCS CONCEPTS • Human-centered computing Interaction design Empirical studies in interaction design

Additional Keywords and Phrases: Meditation technology, Embodied metaphors, Haptics, Thermal feedback ACM Reference Format:

First Author's Name, Initials, and Last Name, Second Author's Name, Initials, and Last Name, and Third Author's Name, Initials, and Last Name. 2018. The Title of the Paper: ACM Conference Proceedings Manuscript Submission Template: This is the subtitle of the paper, this document both explains and embodies the submission format for authors using Word. In Woodstock '18: ACM Symposium on Neural Gaze Detection, June 03-05, 2018, Woodstock, NY. ACM, New York, NY, USA, 10 pages. NOTE: This block will be automatically generated when manuscripts are processed after acceptance.

\section{INTRODUCTION}

The last two decades have been marked by a growing $\mathrm{HCl}$ interest in first-person experiences, embodiment, and wellness as key aspects of the third wave $\mathrm{HCl}$ agenda [12] and its somatic turn [50]. The focus on bodily experiences, felt sense [13], affective interaction [31] or somaesthetics [79] has paved the way towards designing for richer and more expressive user experiences able to support engagement, reflection, and selfawareness; particularly for wellbeing and mental health like in the case of meditation tools [73, 89].

Meditation is a mind-body practice that relies on the regulation of attention to support the non-judgmental awareness of the ongoing present experience both internal such as sensations, thoughts or feelings, and external to limit mind-wandering and enabling being mindful [22, 23, 41]. The importance of paying attention to the bodily sensations has been long acknowledged in meditation and more broadly in mind-body practices [23, $27,29,88$ ] to help regulate emotions or attention [53]. 

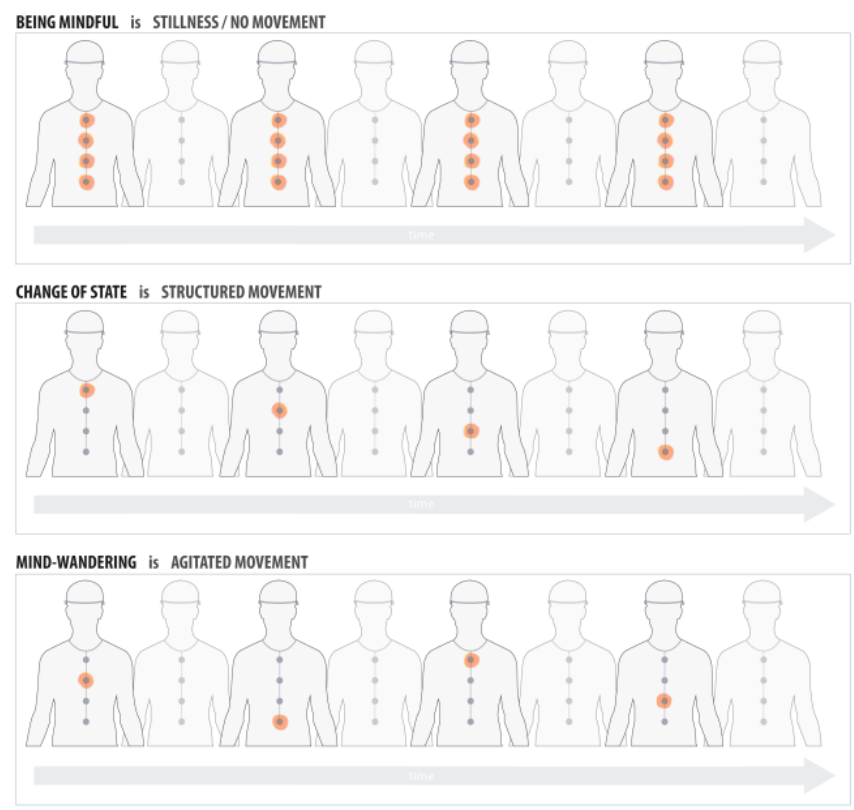

Figure 1. Thermal patterns based on embodied metaphors for meditation provided by WarmMind: being mindful is stillness / no movement (top), change of state is structured movement (middle), and mind-wandering is agitated movement (bottom).

The advent of wearable biosensors and actuators has provided opportunities to push forward research in this space by capturing and communicating bodily experiences back to users, to support reflection and sensemaking for both emotional awareness and regulation as well as attention regulation. A key challenge here is mapping bodily experiences to the interface modality and its content design [1, 72]. Unlike affective technologies for emotional awareness, the design of such mapping of bodily experiences to interface modality and content is even more challenging when designing meditation technologies where the aim is to support attention regulation. There is a threefold reason for this. First, being mindful and mind-wandering states involved in meditation training are less accessible particularly for novice meditations [19, 91] when compared to changes in physiological arousal accompanying, for instance, emotional responses to stress. Second, the mapping has to be subtle not to distract attention from the meditation itself [75]; and third, it has to strike the right balance between ambiguity and clarity, so that the mapping is understood without demanding focus of attention or engendering self-judgment when mind-wandering states are identified.

Despite these challenges, the meditation's benefits for wellbeing [13] have led to a growing $\mathrm{HCl}$ interest in meditation [41]. A common approach is mapping brain activity captured by electroencephalography (EEG) data to visual and aural modalities, generally with being mindful mapped as metaphorically "high" and mindwandering states as metaphorically "low" in terms of spatial orientation or sound pitch. With a few exceptions [75], however, the design of such mappings of bodily experiences to interface modality and content has been limitedly described or their rationale reflected on, and limited efforts have been made to link these mappings to the embodied metaphors underpinning them. So, despite the growing research in biofeedback technologies for wellbeing and particularly neurofeedback ones for meditation, the field is yet to develop a theoretically informed framework to support the design of such mappings. 
Our paper addresses this gap, by exploring the value of the contemporary theory of metaphor as one of possible theories informing such framework. We advance a framework for mapping bodily experiences onto interface modality and content informed also by the phenomenology of meditation experience and embodied metaphors of meditation. We have developed and revised the framework through an exploratory study comparing embodied metaphors of three key meditation states: being mindful, mind-wandering, and the changes between them, represented through both external and internal stimuli as aural- and thermalneurofeedback, respectively. If external stimuli have been often explored through visual and aural modalities, internal ones and their role in supporting embodied metaphors have received less attention.

For our study, we have purposefully chosen Muse [46, 57], an increasingly popular commercial brain activity application providing nature-inspired soundscapes mapped to meditation experiences. We designed and implemented WarmMind (Figure 1), a research prototype providing counterpart mappings to Muse's ones, which differ in three important ways: (1) WarmMind targets bodily internal senses, namely thermoception or perception of bodily temperature, rather than external stimuli; (2) it provides discrete, rather than continuous feedback, (3) through thermal patterns whose meaning is more abstract and ambiguous, rather than familiar.

Our contributions are threefold. First, we introduce interoceptive interaction which extends the design space of meditation technologies to interoceptive modalities as outputs for metaphorical representations. Second, we developed WarmMind, a design exemplar illustrating interoceptive interaction through thermoception as an internal bodily sense. Third, we propose an initial framework capturing the tension between metaphors' discoverability and attention regulation underpinned by three qualities of embodied metaphors: sensory stimulation, feedback temporality, and ambiguity.

\section{RELATED WORK}

\subsection{Meditation Technologies: Mappings of Meditation Experiences}

Within $\mathrm{HCl}$, the way in which technology can facilitate the practice of meditation has received growing interest [85]. In this space, many scholars have explored biofeedback (e.g. EDA [82], HRV [69], Respiration [56, 59, 64]) in which internal states are creatively mapped into visualizations [3,64] or sounds [75, 92] as a means to provide tailored, real-time support during meditation. However, the physiological signal most relevant for meditation is brain activity, as it can monitor mental states such as mind-wandering and being mindful in an accurate and non-intrusive manner [85]. Our review identified two classes of meditation technologies. The largest of these classes consists of systems mirroring user's meditation states in real-time through natureinspired representational metaphors in visual or aural modality $[5,6,16,18]$. In the other class, meditation states are mapped through abstract representations such as color properties e.g. hue [65] or opacity [45, 65], as well as spatial metaphors e.g. low vs high pitch binaural beats [75] and central vs peripheral position [36].

As illustrated above, a common characteristic of neurofeedback-based meditation technologies shared by both these classes is the reliance on polarity to represent the two distinct meditation states: being mindful vs mind-wandering. Another important characteristic of most meditation technologies is the common use of external visual or aural interfaces for mapping the metaphorical representations of the two key meditation states. However, this can be problematic given the importance of paying attention to and regulating one's bodily sensations arising during meditation, which is key for this embodied practice [19, 43, 61]. 
Alternative modalities that can support internal representations of meditations states have been less explored. A good candidate here are haptic interfaces. Indeed, findings showed that haptic biofeedback could support awareness of internal bodily experiences, also called interoception [23], and in turn the self-regulation of internal processes [90]. While $\mathrm{HCl}$ work on haptic feedback for meditation technologies is limited [14, 55], useful related work has explored vibrotactile and thermal biofeedback for emotional awareness and regulation $[1,90]$. In this respect, findings indicate that the inherent affective quality of thermal sensations is reflected in abstract and subtle of thermal feedback [40]. Landmark examples in this space [2, 32] suggest that thermal haptic feedback is perceived as a pleasurable yet subtle and unobtrusive form of feedback, in which warmth is mapped to positive sensations while cold to the opposite.

To conclude, most work on mindfulness technologies has focused on either abstract or representational metaphors of both being mindful and mind-wandering states, predominantly through external visual or aural stimuli. In contrast, limited work has explored the metaphorical mapping of meditation states on internal stimuli for instance through thermal actuators and their potential to support meditation, given their subtle and pleasurable qualities.

\subsection{Embodied Metaphors in $\mathrm{HCl}$}

Metaphors have a rich history in $\mathrm{HCl}$ [11] from supporting designers research unfamiliar topics or get inspired by novel associations, to communicate their ideas [70] and ultimately the mental model of systems to users [15]. The theory of conceptual metaphor that builds on the embodiment tenet according to which bodily perception, movement, and experience of the physical world (including gravity and force dynamics) underpin our conceptual and abstract thinking [48]. When such bodily experiences across a range of scenarios get structured as recurrent patterns, they become image or embodied schemata [37]. The grounding in sensorymotor experiences makes image schemata multimodal, which means that they can also be represented in different modalities, the most researched ones being aural, visual, and haptic. Lakoff [48] argued that embodied schemata support abstract thinking, as people build metaphors that extend schemata from the physical to the abstract domain, such as Better is Up, Worse is Down [49]. Metaphors that extend image schemata are called embodied metaphors [8] and emerge through repeated connections of their image schemata with the subjective judgments of the respective abstract domains such as affect or morality [35].

Image schemas and embodied metaphors tend to be intuitive [35] or easily understood, often without the need for words [51]. This quality has been linked to the discoverability of the underpinning mapping of the image schema [51, 70] which can be represented in different modalities. However, some are more ambiguous than others depending on the content being mapped from the source to the target domain [35]. Hence, a main challenge of designing with embodied metaphors is identifying the best content to be mapped and the instantiation of embodied schemata [35]. Among different classifications of metaphors, those relevant for $\mathrm{HCl}$ include a distinction between orientational or spatial metaphors emphasizing bodily orientation through space and time: up-down, front-back, near-far, left-right, and ontological ones underpinned by bodily interactions with physical objects, i.e. desktop metaphor, that can be used to communicate abstract concepts in terms of physical ones [47]. Previous work has suggested that spatial embodied metaphors may be easier to understand [4], particularly those organized along the vertical axis to map abstract concepts such as emotions, although limited empirical work has explored this. 
Apart from the theoretical work outlined above, much of the $\mathrm{HCl}$ applied work on embodied metaphors took place in tangible interaction research, for instance, to explore the bodily understanding of abstract musical concepts [7] and different states of environment preservation and food production [51]. In such cases, the embodied mapping (i.e. volume was mapped to activity reflected in moving arms, stomping feet through more is loud and less is quite) was more intuitive and easily understood compared to the non-embodied one. Furthermore, metaphoric mapping appeared to support performance but lacked discoverability, i.e. it was understood at a subconscious level but probably without forming an explicit understanding of it [51].

To conclude, most $\mathrm{HCl}$ work on embodied metaphors emphasized their support for intuitive understanding of abstract concepts such as sound concepts or social justice through bodily movement. Such work highlighted metaphors' qualities such as polarity and easier enactment than verbalization. The embodied metaphors were elicited from both experts or users to discover the best physical movements representing abstract concepts, and evaluated through studies where the enactment of movement was used to control changes in abstract concepts represented through sound or images. There has been however less exploration of the different modalities for the same abstract concepts including those about interoceptive senses such as thermoception. Moreover, tangible interaction work on embodied metaphors explored the physical to abstract mapping, with image schemata usually enacted through bodily movement. The exploration of embodied metaphors in mindfulness technologies unpacked metaphors highlighting the body to mind mappings that link bodily experiences to one's mental states instead of abstract concepts [6], enacted though attention regulation to external or internal stimuli. The aim of the two domains are different: body and movement to interact with tangible artifacts vs to train attention regulation. Hence, metaphors' properties such as polarity, multimodality [35], and discoverability [4] could play different roles in other classes of technologies such as those challenging habits, supporting reflection [34] or in our case, supporting mindfulness training.

\section{EXPLORING EMBODIED METAPHORS OF MEDITATION STATES}

In Focused Attention Meditation (FAM) [23], the focus of attention is on an explicit, clear and unwavering (calm and stable) [60] stimulus such as candlelight, mantra sound, or one's breath [25]. If the stimulus is not visual, a common instruction is that meditator's eyes are closed to limit potentially distracting external stimulation. Although the explicit stimulus can be either external or internal, most work on meditation technologies has focused on external stimuli, commonly images or sounds.

In this section we describe the preliminary work informing our design rationale to explore thermoceptionbased embodied metaphors for meditation states, and their comparison with counterpart sound-based metaphors. We agree with the view that identifying appropriate metaphors is not trivial, and can benefit from multiple sources [35]. For the exploration of interoceptive-based metaphors, we triangulated three methods which are detailed below: review of $\mathrm{HCl}$ work on embodied schemata for mindfulness technologies (section 2.1), work on cognitive linguistics on metaphors related to meditative practices, and the phenomenology of meditation.

\subsection{Cognitive Linguistics Research on Meditation Metaphors}

The exploration of meditation in cognitive linguistics has looked at corpus of text often from Buddhism discourse to explore the different embodied metaphors, common ones being those involving water and movement. Ontological dynamic metaphors include those of the ocean and its waves, for instance, waterscapes whose stillness relates to calm mind or being mindful states, and wind-induced waves or agitation which relates to troubled mind or mind-wandering states $[42,80]$. Another common metaphor is that of meditation is a journey, 
and the meditator is an observer of one's body, thoughts, sensations or feelings [80]. Despite their richness and consistency, these metaphors capture less the lived experience of meditation, which we briefly review below.

\subsection{Phenomenology of Lived Experience of Meditation}

The lived experience of meditation has been explored mostly in mindfulness research and more recently in $\mathrm{HCl}$. In novice meditators, Basnett [10] found that their most common subjective experiences included bodily sensations such as tingles, light/heaviness, a quarter reported feelings of warm, and two reported awareness of heart-beats. Another study of meditators' phenomenological experience explored across four practices (i.e. breathing, body scan, loving-kindness and observing-thought) has shown that and meditators across all practices experienced increased feelings of warmth and interoceptive awareness [44]; particularly in the upper body such as chest and throat [61].

Recent $\mathrm{HCl}$ work also explored the bodily experiences associated with key meditation states [19]. Findings abound in metaphorical language indicating that meditation is experienced as a sensorial journey consisting of five key stages with specific bodily experiences: grounding is downwards movement, becoming mindful is upwards movement and warmth in the belly, being mindful is being in balance, and mind-wandering is loss of bodily awareness. Expert meditators suggested the value of recreating such bodily sensations through downward movement for mind-wandering, or of upwards movement to facilitate being mindful [19].

The above outcomes complement previous work on the importance of bodily sensation in meditation [23, 27, 29,88 ]. They also provide additional phenomenological evidence for the value of designing interoceptive stimuli to support bodily awareness, and in particular novices' bodily awareness through FAM. These findings also shed light on specific bodily sensations characterizing each meditations stage and the involvement of two interoceptive senses [67], namely breathing and thermoception [76], alongside metaphors related to movement. Compared to breathing, thermoception has been less explored in mindfulness technologies, presenting thus the opportunity to open up this design space.

To conclude, findings on the lived experience of meditation identified metaphors involving bodily sensations as emphasizing warmth and movement, hence more suitable to be perceived through interoceptive senses such as thermoception (Table 1). In contrast, findings on cognitive linguistics indicated metaphors involving external objects and processes such as water and movement that appear more representational, hence better suited to be communicated through external senses such as vision or sound. The identified metaphors from both sources offer an exciting space for design exploration as they share movement as a process, but also warmth and water respectively which can be communicated to internal and external senses, respectively.

\subsection{Analysis of Muse: Audio-based Metaphors}

Our aim to explore novel meditation technologies leveraging thermoception can benefit from comparison with traditional interfaces leveraging external senses. As vision is not required for thermoception, and in the interest of limiting the overall stimulation for the FAM that we aim to support, we decided to explore also aural meditation interfaces. Muse [57] is a wearable headband monitoring brain activity through 5 cutaneous channel electrodes which infer real-time meditation states. Its app provides a sound-based interface whose default settings is a rainforest soundscape, leveraging thus water and movement-based metaphors. Previous work has shown the validity of Muse headband measuring event-related brain potentials $[46,66]$ and meditation states $[18,46]$. 


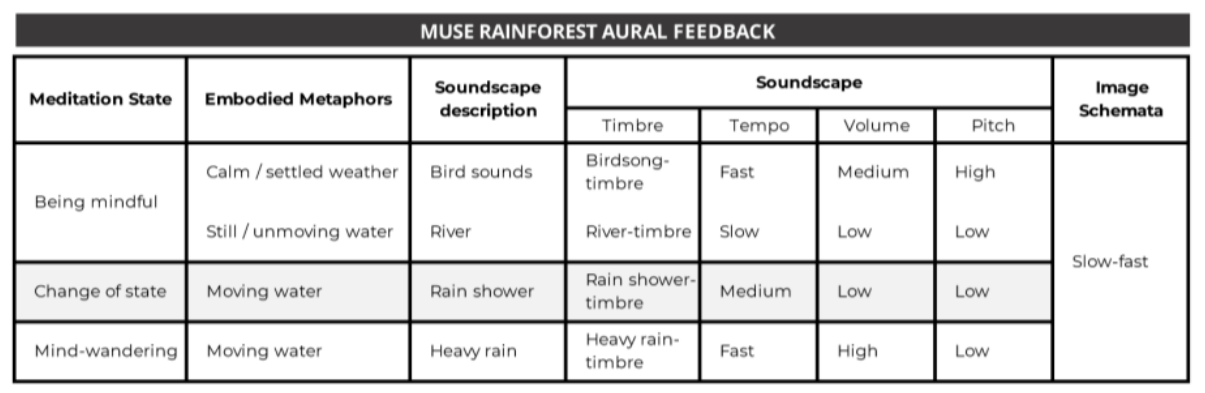

\begin{tabular}{|l|l|l|l|l|l|l|}
\hline \multicolumn{9}{|c|}{ WARMMIND THERMAL FEEDBACK } \\
\hline Meditation State & $\begin{array}{c}\text { Embodied } \\
\text { Metaphor 1 }\end{array}$ & $\begin{array}{c}\text { Embodied } \\
\text { Metaphor 2 }\end{array}$ & Thermal pattern description & \multicolumn{2}{|c|}{ Thermal stimuli } & $\begin{array}{c}\text { Image } \\
\text { Schemata }\end{array}$ \\
\cline { 4 - 6 } Being mindful & No movement & $\begin{array}{l}\text { Radiating } \\
\text { warmth }\end{array}$ & $\begin{array}{l}\text { Simultaneous activation of all } \\
\text { heat actuators (subtle pulsating } \\
\text { sensation) }\end{array}$ & Structured & Medium & Intensity \\
\hline Change of state & Movement & Less warm & $\begin{array}{l}\text { Ordered activation of heat } \\
\text { actuators to (downwards } \\
\text { movement from chest to belly) }\end{array}$ & Structured & Low & Structured - \\
unstructured \\
\hline $\begin{array}{l}\text { Mind- } \\
\text { wandering }\end{array}$ & Movement & Less warm & $\begin{array}{l}\text { Random activation of heat } \\
\text { actuators (random movement) }\end{array}$ & Unstructured & Low & \\
\hline
\end{tabular}

Table 1. Top: Muse embodied metaphors for each meditation state, their soundscape descriptions, associated soundscape properties, and image schemata. Bottom: WarmMind design of embodied metaphors for each meditation state, their soundscape descriptions, associated sound properties, and image schemata.

In this section we describe the audio-neurofeedback provided by Muse, emphasizing our analysis of its associated embodied metaphors and image schemata underpinning them, as well as the sound properties and how they vary among the meditation states. Muse app provides a continuous forest soundscape adapting in real-time to the current mental state. It instructs users to focus on breathing, with their eyes closed while listening to the soundscape. The app identifies three meditation states: being mindful (when the person is fully focused and relaxed), mind-wandering (distracted mind when attention is fluctuating), and change from one to another (moments in which attention is not fluctuating, but there is no deep focus present). Table 1 (top) shows the embodied metaphors for each state, soundscape descriptions, and associated sound properties we identified.

\section{DESIGNING WARMMIND: THERMOCEPTION-BASED METAPHORS}

The design of WarmMind prototype was intended to extend the design space of meditation technologies through interoception-based interaction. Albeit, in doing so, we also wanted to better understand in what fundamental ways such design may differ from the one for exteroception-based interaction. Hence, our intention to ensure a comparable design of WarmMind to that of Muse app, was also grounded in the movement-based metaphors underpinning them both. Thermal sensations can be generated through heat pads which is a novel material for meditation technologies, both for us as designers and meditators. Given the highly exploratory nature of our work, the emphasis was less on building an effective meditation aid but rather on better understanding the dimensions and qualities of designing for thermoception modality. Thus, we allowed ourselves to experiment, compare and purposefully stretch some dimensions of the design space to increase their impact on meditation experience so that we learn more about them. Such understanding could then lay the foundation for designing effective thermoception-based meditation technologies, and interoceptive ones more broadly. 
The rationale for this less explored interceptive sense, whose receptors respond to temperature changes within one's body [84], is to better support novice meditators' focus of attention on the inner experience of meditation and their associated bodily experiences, rather than on external visual or aural stimuli as most mediation technologies have explored. This further aligns with the aim to limit unnecessary stimulation and to allow for the focus of attention on breathing to be sustained, rather than being distracted by any other stimuli, thermal ones included. This meant a clear choice from the start for a rather subtle form of neurofeedback, unlike the continuous one in Muse app. Thus, we explored discrete feedback, only when the transition points between the three states are experienced: for instance from being mindful to change state, or from change to mindwandering state.

\subsection{Integration of Movement- and Warmth-based Metaphors}

Movement-based metaphors consistently link being mindful to stillness, and mind-wandering to movement or agitation [80]. Thus in designing the metaphor for the mind-wandering state, we started exploring the idea of a property that can capture chaotic patterns, and such property is rhythm, associated with structured-unstructured image schemata [8]. It has been previously explored in tangible interaction research through metaphors mapping the abstract concept of sound's rhythm to movement's structure [8]. Thus, we decided to explore the metaphor mind-wandering is chaotic movement. Both upwards and downwards movements are vertical movements experienced during meditation on the front upper body [19], e.g. when following the breath moving from the chest down to the belly, and back up [41]. Thus, we started thinking about the placement of heat actuators on a vertical line from the chest to the belly.

As proximal sense, thermoception will be supported through heat actuators whose placement on the body needs to be carefully considered. To design for the vertical movement and its physical form, we draw inspiration from the traditional meditation beads necklaces. Such necklaces consist of 108 beads placed on a string that are used to help the mind focus on meditation, for example, by repeating a mantra for each bead [68]. In order to create a vertical movement along a vertical axis of the front upper body, the necklace would need to consist of at least three colinear beads (or heat actuators). With the necklace concept in mind, we turned our attention back to the metaphor mind-wandering is chaotic/unstructured movement. However, we found that only three points may not be sufficient to create a feeling of chaotic movement, and to increase the expressiveness of the mind-wandering metaphor, we considered a necklace consisting of four beads on a vertical string. Therefore, we designed a thermal pattern that randomly warms up one actuator at the time to better communicate a sensation of randomness or lack of pattern, as described in the next section.

\subsection{Somadesign Exploration of Movement- and Warmth-based Metaphors}

We employed a soma design approach [33] to explore our first-person bodily experiences while interacting with the WarmMind prototype, a design exemplar used to articulate design knowledge and push the dimensions in this space [74]. This first-hand exploration was shaped by thermal actuators and our interaction with them to experience the temporal unfolding of temperature changes on our bodies, our understanding of thermal patterns, and how they can be better designed for increased expressiveness and communicative power. This exploration involved a group of 3 researchers (2 female, 1 male) meeting over several weeks to experiment with different sizes and placements of the heating pads on the body, their temperature intensity, duration, and pattern orchestration. 


\subsubsection{Exploration of Thermal Stimuli for Meditation}

Our exploration was guided by key aspects underpinning thermoception. Neutral thermal state is the range at which human skin usually rests (usually $28^{\circ} \mathrm{C}-40^{\circ} \mathrm{C}$ ), and within which thermal changes are difficult to detect [38]. This range is stable within an individual but can differ across individuals, i.e., $30-36^{\circ} \mathrm{C}, 28-34^{\circ} \mathrm{C}$. Just noticeable differences is the minimum amount of change from current skin temperature that is required to be detectable $[9,17,86]$. These two parameters were initially adjusted for each user by calibrating each of the four actuators to ensure they were all noticeable and safe. For convenience, we started exploring the temperature intensity on more accessible body parts such as our arms and found out that the feeling of comfortable warmth meant different temperatures for each of us. Then we moved to exploring on our upper body, starting with one actuator at the time. We particularly looked at the temperature range which upper body would detect as pleasurable, without being too subtle or too hot. We found that nice warmth was perceived around $40^{\circ} \mathrm{C}$ when applied for 6 seconds (Error! Reference source not found., left), although individual differences emerged with comfortable temperature ranging from $35^{\circ} \mathrm{C}$ to $45^{\circ} \mathrm{C}$. Because a narrow temperature range made it more difficult to distinguish between different temperatures on the skin for which warmth felt pleasurable, we decided to keep this full temperature range consistent for each actuator and to generate the thermal patterns by altering their temporal aspects.

\subsubsection{Creating Movement with Warmth: Location, Size and Duration of Actuations}

With respect of bodily location of the actuations, we felt how different areas of our upper body responded differently to warmth: chest was by far the most sensitive one, while belly the least. For instance, temperature intensity over $45^{\circ} \mathrm{C}$, was felt as a sensation of stinging on the chest, and as a diffuse feeling of warmth on the belly. Such felt sensations provided us with a more nuanced understanding of previous findings on the increased thermal sensitivity of the upper body [39], and sensitized us to the importance of personalizing the temperature intensity and particularly for each one of the four heat pads and their bodily placements.

Regarding the size of the heating pads, we started with actuators of circular shapes of $5 \mathrm{~cm} \times 5 \mathrm{~cm}$, which felt comfortable on the skin. However, along the vertical string on which we placed them, such rather large pads could fit at most $2 \mathrm{~cm}$ apart, which led to the feeling of warmth radiating around them in almost contiguous areas. This led to the entire upper body feeling warm, with limited ability to recognize the location of each actuator. In this way, we felt on our bodies the property of the skin to summate the intensity of warmth over space and the rather poor spatial resolution of thermal interfaces [62]. This is a challenge limiting the ability to recognize the thermal patterns and to distinguish them from each other, which we addressed by exploring smaller sizes for the heat pads. We found that circular-shape $1.5 \mathrm{~cm} \times 1.5 \mathrm{~cm}$ placed at least $2 \mathrm{~cm}$ apart can be individually perceived without blending into each other (Figure 2, left).

When it came to explore thermal patterns, we found two temporal aspects that mattered most, namely the duration of actuation (long enough to be noticed, and not too long to stop being pleasurable), and time between consecutive actuations. We found that the actuation was unnoticeable when applied for less than 4-seconds and uncomfortable when lasted longer than 8 seconds, consistent with previous findings on on-skin warmth perception [40]. Based on this, we designed each actuation to be on for 6 seconds, and have 4-seconds off before the start of the next actuation to allow for the warmth to dissipate. This actuation was the building block for each of the three thermal patterns, that were formed of 4 actuations to make sure that all actuators warmed 
up at least once within each pattern. Further, each pattern was designed to be felt distinct from the other, whilst keeping the same aesthetic quality of subtleness.

\subsubsection{Metaphorical Representation of Meditation States with Thermal Patterns}

Movement-based metaphors consistently link being mindful to stillness, and mind-wandering to movement or agitation [80]. We now describe the metaphors used (Table 1, bottom) and the first-person experience to design the thermal patterns mapping meditation states (Figure 2, right).

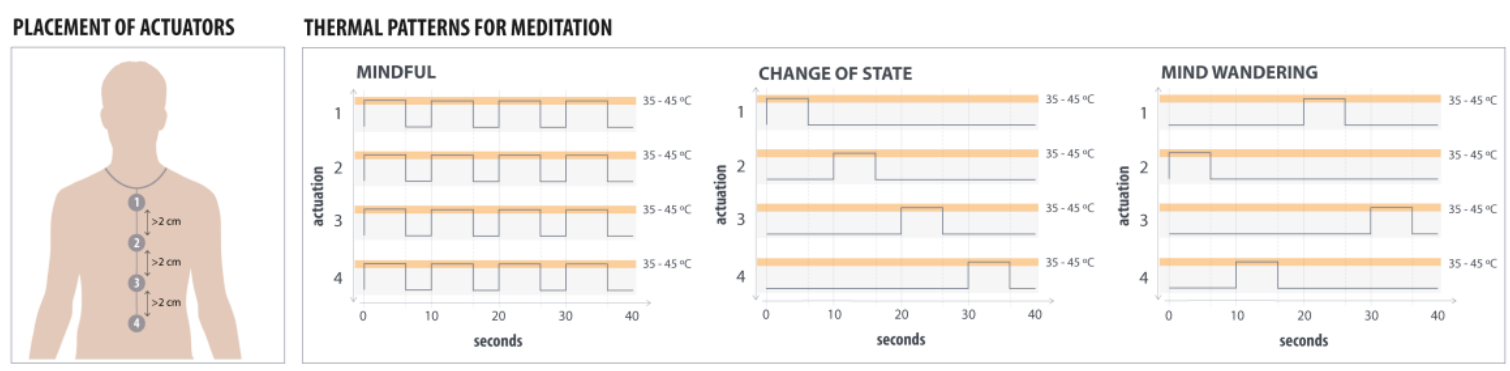

Figure 2. Diagrams of the WarmMind prototype's thermal patterns designed to map meditation states of being mindful (left), mind-wandering (right), and change of state (center).

On the one hand, the being mindful state was mapped to 4 simultaneous activations of all heat actuators as a subtle pulsating sensation. We implemented this as shown in Figure 2 right, by simultaneous activations of all actuators for 6 seconds at their specific personalized temperature, repeated 4 times after 4-second intervals. We aimed to create no vertical movement so that stillness is properly communicated. For increased expressiveness, we considered however a pulsating pattern of each actuation, similar to the metaphor of beating heart. This pulsating pattern is also consistent with previous work, that found that in when being mindful, people experience a sensation of warmth around the heart area, similar to a beating heart [19, 43]. When experimenting with this pattern, it felt like waves of warmth coming and going at a slow beating pace. We liked this pattern as it felt like augmenting the natural movement of breathing on the upper body.

On the other hand, the mind-wandering state (Figure 2, right) was mapped to random activation of the 4 actuators, with each of them being activated only once in random order at their specific personalized temperature. Although during meditation we found it difficult to identify the order of the actuations being activated, this pattern generated a nice and subtly agitated warmth sensation moving on the upper body. It also felt very distinct to the pulsating sensation of the being mindful state, which was important to us, since being mindful and mind-wandering can be conceptualized as being polar, each one placed on the ends of a continuum.

Finally, the change of state was mapped to ordered activation of heat actuators to represent a downwards movement from chest to belly. For this, we actuated each heat pad for 6 seconds, with the actuation starting 4seconds after the heat pad, which was placed immediately above on the vertical string, has finished its activation (Figure 2 right). We experimented with different vertical movements to link the states of being mindful and mindwandering, and found it difficult to recognize the direction of movement as up or down. This may be because of the less thermoception accuracy on the belly area, and the dissipation of warmth around the actuation. Therefore, we chose just one direction of movement: to start on the chest and move down to the belly as we found the chest to be the more sensitive area and, this way, the sensation moved from being more intense to 
more diffused. We found this to be a nice middle point between the pulsating sensation associated with being mindful and the random actuation associated with mind-wandering, by moving attention to the vertical line within the front of the upper body in a structured manner.

\subsection{WarmMind Prototype}

We now describe the WarmMind prototype we developed to better understand the above metaphors and how they can be instantiated in design. Informed by our initial metaphor exploration outlined above, the WarmMind prototype is an on-body interface in the form of a necklace for providing the thermal patterns during meditation. The necklace has four actuators made of Nichrome wire heating pads to be placed on the upper body from the chest to the navel. Nichrome wire heating pads are low-power, inexpensive, and malleable material, which get warm when powered with a 5VDC. To support experimentation with the actuators we developed a tangible interface consisting of an Arduino baseboard microcontroller, 4 MOSFET modules, and 4 LEDs -one for each heating pad-, as well as a joystick to select, control and change in real-time the thermal patterns.

\section{METHODOLOGY}

Our exploratory study aimed to compare the two modalities: aural and haptic thermal neurofeedback provided by Muse app and WarmMind prototype, respectively. We run individual sessions with our 10 participants, each session lasting about one hour. We employed a reverse counterbalancing within-subject design, ensuring that all participants were exposed to both feedback modalities, as detailed in the User Study section below.

\subsection{Participants}

We recruited 10 participants ( 5 female 5 male, range 19 to 25 years old, average age 21 ) through university mailing lists and notices posted in campus. A total of 4 participants had previous experience with meditation, of which 2 started the study with the haptic condition and the other 2 with the audio neurofeedback condition.

\subsection{User Study}

Upon arrival, each participant was given the study description and a short presentation introducing the practice of FAM and neurofeedback, and the two systems: WarmMind prototype and Muse [57]. The study started after participants signed the consent form and consisted of four parts as detailed below.

\subsubsection{Prototype Setup and Calibration}

The first step was to set up the WarmMind prototype. To respect privacy, participants were instructed on how to place the actuators and were left by themselves in the room to set it up. Then, a calibration test was conducted to adjust the temperature range of each actuator so they were both noticeable and comfortable. This consisted of turning on each individual heating pad at the lowest intensity for 6 seconds, and then turning it off for 4seconds. Participants were asked to say when they felt any warmth coming from the pads. The customized thermal range for each actuator (full range across the study sample was $35{ }^{\circ} \mathrm{C}$ to $45{ }^{\circ} \mathrm{C}$ ) was saved for the duration of the study. This was also an important step to ensure safety as the thermal threshold on different body locations can vary between people [86].

\subsubsection{Exploring Meditation with Aural and Thermal-based Neurofeedback}

For both feedback modalities, participants were asked to meditate for 5 minutes, with their eyes closed while sitting comfortably on a chair. They were also instructed to pay attention to their breath, slowly filling their chest 
and belly, facilitated by the common FAM posture of placing one hand on top of the chest, and another on the belly which encourages deep,controlled breathing [30]. Between meditation sessions, participants had a 5minute break to diminish the carryover effect.

The aural feedback by the Muse's app was played through Audio-Technica M40X studio grade headphones. The thermal patterns were provided through the WarmMind prototype, as described in the previous section, and the integration with the headband was by proxy through a Wizard of $\mathrm{Oz}$ approach. The researcher was listening to the participants' Muse [57] soundscape in real-time and provided accordingly the corresponding thermal feedback. To control for the effect of the mere presence of different devices and to ensure consistent experience regardless of feedback modality, the Muse headband and app were used in both conditions, alongside the headphones and the WarmMind prototype. Since the use of Muse headband in conjunction with Muse app prevents access to real-time EEG data, we used self-report measures of state mindfulness (not trait), before and after each meditation session. Mindful Attention Awareness Scale (MAAS) for state [87]. This is a standardized instrument widely used to assess state mindfulness, as a receptive state of mind in which attention, informed by a sensitive awareness of what is occurring in the present, simply observes what is taking place [13].

\subsubsection{Semi-structured interview}

The semi-structured interviews aimed to explore users' perception of the two neurofeedback modalities, their understanding of the two mappings of meditation states to the soundscape and thermal patterns, and their impact on meditation experience. We asked whether they preferred audio or thermal condition and why, as well as what were the main benefits and challenges in each condition. We further asked about their perception of meditation states when meditating with audio and thermal neurofeedback, by enquiring about how each condition impacted on the recognition of e.g. being mindful and mind-wandering moments. We also asked about the experiential qualities of the thermal patterns, the on-body location of the actuations, whether they could identify distinct thermal patterns, and potential integration of this feedback modality in meditation technologies.

\subsection{Data Analysis}

Interviews were fully transcribed and coded, following a hybrid coding approach [24] using the Atlas.ti8 software for qualitative analysis. The development of the coding scheme was an iterative process involving all authors. Drawing upon a conceptual framework developed from prior work on meditation [19, 58, 71], the initial codes included the main strategies for meditation, key meditation stages, and the physical sensations associated with meditation, as well as qualities of embodied metaphors (e.g. polarity, discoverability, ambiguity, discrete, continuous). Then, we refined the scheme to include codes that emerged from the interviews such as new qualities of embodied metaphors and about the meditation experience, such as: main focus of attention (i.e. internal / external), support or hindering for attention regulation (i.e. ambiguous / familiar, subtle / rich), and maintaining non-judgmental stance (i.e. abstract / representational).

\section{FINDINGS}

We now detail the qualitative analysis of the semi-structured interviews, organized in four main themes: (1) the main focus of attention, (2) recognition and understanding of the embodied metaphors for meditation states, (3) supporting regulation of attention, and (4) supporting non-judgmental attitude during meditation. 


\subsection{Focus of Attention}

\subsubsection{External: The Soundscape Becomes the Focus of Attention with the Muse App}

An important finding regarding Muse App is that most participants (7) mentioned that the rather engaging quality of the soundscapes attracted their attention. This was usually due to the representational feedback and immediate communication in changes of meditation states: "I did find that, like, with the sound, it would do something immediately and I would be like 'okay I know what caused that to happen" (P9). Because of this quality, participants mainly focused and maintained their attention on the feedback provided: "I was focusing on the sound, the sound was really helpful thing when I hear birds I was trying to just maintain as long as I can. " (P2). However, most participants (8) did not like the fact that the soundscape became the only focus of attention as it distracted them from their meditation experience, as shown in this illustrative quotes: "[the soundscape] it was just a bit too direct I think, and I don't know, hearing it made me a bit distracted" (P7), "the noises were annoying, when it started raining more heavily I got annoyed, so it was hard to focus again. [...] although the sound was clearer [easier to understand the mapping] [...] I don't think the feedback helped me" (P8). Having the focus of attention on the soundscape meant that the regulation of attention back to the breathing was not direct, but mediated by the sound: "my brain would stop [engaging with distracting thoughts], and then my mind would be like 'Okay, I'm focusing on my breath, I'm doing something right'. And then I would lose my focus and the rain would start again" (6). As illustrated in this quote, with the Muse app the breathing becomes a secondary focus of attention to the soundscape.

Another highlighted aspect of Muse App, mentioned by 5 participants was how the audio feedback allowed them to disengage from external stimuli such as environmental noise and focus on the soundscape: "I feel I liked sound, because sound is more isolating. Not only do you focus on what you are listening to, but also tune out everything else around you" (P4). Other 3 participants highlighted that the soundscape allowed them to also disengage with internal stimuli, such as their thoughts: "because of the sound of the water, you don't focus on your own thoughts so much, you isolate more [...] it made you unaware of everything that's around you " (P5).

\subsubsection{Internal: The Body Becomes the Focus of Attention with the WarmMind Prototype}

Findings indicate that whilst audio feedback was perceived as an external stimulus for the attention, both engaging or stimulating but mostly distracting, the warmth patterns were sensed as internally, coming from inside the body, allowing participants (7) to focus attention inwards on their personal meditation experience: "I think the audio is more about the technology, and the heat is more about coming back to yourself [...] and focusing on your body. [...] It's kind of like turning on your all different senses" (P1). This illustrative quote indicates that thermoception feedback supports increased awareness of one's senses, which contrasts with the common experience of isolation enabled by the audio feedback. As a result, with the thermal patterns, the body takes a central role, becoming the focus of attention during the meditation: "I feel like [WarmMind] also makes you more aware of your body. Because we don't usually focus on that type of change - [...] basically the temperature of different parts of your body; we're not really focused on that. So I feel like this also helps you build awareness in that aspect' (P5). This quote and the following one also illustrate how the thermal actuation is perceived as internal to the body rather than from an external source i.e. our prototype: "it felt like it's quite a natural thing" (P9). An important consequence of the internal focus of attention is the direct access to the breathing pattern felt within the body, without being externally mediated by sound, like in the Muse app. As mentioned by 7 participants, the thermal feedback allowed for the deep breathing to be maintained within the focus of attention: "it's quite good in the torso, especially with the breathing out to the breathing on the top" (P3). 


\subsection{Recognizing and Understanding the Metaphorical Mappings}

\subsubsection{Familiar Sounds Facilitate Identification of Meditation States}

Regarding the identification of the distinct metaphors representing different meditation states, 6 participants identified three main sounds: "there was that kind of heavy rain, light rain, birdsong" (P3). which was particularly useful to recognize the metaphors. Findings particularly show $d$ that the moments of being mindful and mindwandering were the easiest ones to become aware of and understand during meditation: "when I sort of started letting my mind go, I could start hearing the rain stop and the birds singing" (P10). As illustrated by this quote, all participants easily understood the metaphor of rain for mind-wandering, and no rain and birds singing for when being mindful. Nevertheless, although the change of state metaphor was also usually identified: "the water was quieter for a long periods" (P5), it seems that it was less easier to map when compared to the other two: "the actual different sounds for being not concentrated I didn't quite get" (P3). To summarize, the two polar states were easier to map because the heavy rain was higher in tempo and volume compared to light rain, which also had new distinctive sounds of birds singing at high pitch.

\subsubsection{Thermal Ambiguity Difficults Differentiation of Patterns}

Concerning the WarmMind prototype, findings indicate that the perceived ambiguity of the warmth patterns made it difficult to understand their mapping to meditation states: "I couldn't really link what I was thinking with what the feedback [thermal pattern] was giving" (P9). Thermal patterns were more difficult to differentiate as participants could not immediately recognize which actuator was warming up at the time: "I didn't really distinguish like 'is it the middle one, the third one, the fourth one, or the first one; which is heating?"' (P2). However, participants distinguished between a full warmth (i.e. being mindful) and a random pattern (i.e. mindwandering): "the pattern was kind of noticeable when my mind was wandering. It was absence of the same warmth as when I was concentrated" (P3). As with the soundscape, the change of state metaphor was not as clearly understood compared to the two states of being mindful and mind-wandering. Besides, 6 participants identified changes in thermoception as something that requires bringing back attention to the breathing, without being able to discriminate its meaning: "I was only really paying attention to this [heat] and the breathing" (P9).

\subsection{Supporting the Regulation of Attention}

\subsubsection{Rich Stimulation Hinders Attention Regulation}

An important finding reported by most participants was that, although the audio-based feedback allowed them to easily identify their meditation states, they also struggled to regulate their attention in order to maintain it: "although the sound was clearer [easier to understand the mapping] [...] I don't think the feedback helped me, because when I understood that I was doing good, I got back to the start and lost focus" (P8). Findings indicate that most participants found the regulation of attention with the aural feedback challenging as the soundscape was perceived as distracting due to three main issues.

First, the soundscape was perceived as intense, particularly at moments of being mindful and mindwandering: "I feel like [audio] is also more distracting because it's more intense [...] With the sound, I had a problem where I started drifting off and I heard the sound was louder. So I tried to go back to make it quiet, but I was focusing on it too much" (P5). As described in this quote, participants perceived the volume of the feedback to increase or becoming louder as they tried to focus their attention to get back to being mindful. Second is the continuous audio feedback meant that each change from one meditation state to another was mapped through the changes in the soundscape. However, such changes in meditation states can occur rather 
frequently, particularly in novice meditators, and thus, the continuous mapping may lead to fast changes which can hinder focus of attention: "I found the sounds to be a bit maybe too disturbing the way they were changing [...] it was it basically too rapid" (P10). Finally, the soundscape was also found to be rather sensorially rich, or overwhelming: "the sound just overtook all my senses" (P4). Together, intensity, rapid continuous change, and sensorial richness made the soundscape mappings both enjoyable and easier to identify albeit distracting.

\subsubsection{Subtle Warmth Calls for Bringing Attention Back to the Body}

A key outcome is that thermal feedback allowed participants to keep bringing the attention back to bodily sensations and breathing. In turn, this facilitated disengaging from distracting thoughts and achieving a being mindful state: "I couldn't really tell [the patterns]. It was there, and I didn't really pay more attention to it, as I was focusing on my breathing, and my mind, rather than focus on what feedback I'm getting that makes sense, which sort of was just in the background" (P10). This is a significant finding indicating that although the metaphorical representations of meditation states with thermal patterns were more difficult to understand than aural ones, the thermal patterns better facilitated the regulation of attention during meditation. It particularly illustrates the subtle, almost peripheral presence of the thermal neurofeedback, which does not distract, nor engage attention away from meditation, albeit implicitly supported the focus of attention on breathing: "it's [the heat] quite good in the torso as well, especially with the breathing out from the bottom [belly] to the breathing on the top [chest]" (P3). The fact that such support was perceived by participants with and without previous experience with meditation is particularly important. Indeed, they both reported that thermal patterns fostered bringing attention back to the body, despite the fact that interoceptive awareness or awareness of bodily sensations is a skill that develops in time: "it was just like, 'oh its warm, so I have to come back to myself" (P1). Therefore, the embodied qualities and openness to interpretation of the thermal feedback were found to facilitate the regulation of attention at one's pace, whilst subtly suggesting to bring the attention back to the body.

Findings show that all participants perceived the warmth as a subtle form of interaction: "I feel like [the audio] it's also more distracting because it's more intense, whereas the heat was more subtle and just felt like [the warmth] was suggesting, and the water was more of a guide like hard guiding you" (P5). As illustrated by this quote, most participants felt like the thermal patterns were not as intense as the soundscape and allowed them to re-focus their attention without overtaking or overwhelming their senses: "I really liked that it does get your attention but it wasn't like it was distracting" (P9). Interestingly, if aural patterns were at times perceived as too intense, thermal ones were perceived by 6 participants as being too subtle: "I like the heat because it was subtle. [...] Although the heat might have been a bit too subtle maybe" (P4).

For the results of the MAAS questionnaire, we run a paired t-test to compare the effects on the state mindfulness, when meditating for 5 minutes with thermal feedback compared to an auditory soundscape. Although there was an increase in the state mindfulness for both conditions when compared to baseline (increase of 0.3 on average with SD $=3.9$ for the thermal, increase of 1.4 on average with SD $=3.5$ for the audio), it was not statistically significant.

\subsection{Metaphors' Expressiveness Fosters Judgmental Attitude}

As described above, the rainforest soundscape provided by the Muse's apps was perceived as easy to understand and particularly expressive due to different sound timbres (rain, river, birds), decreasing volume and tempo level from mind-wandering to being mindful, and increased pitch associated with birdsong in the being mindful state. Nevertheless, this was found to be a negative aspect of the audio condition for two main 
reasons. First, participants found the metaphors for being mindful and mind-wandering states too representational and affect-charged. For instance, participants were familiar with the metaphors used and linked the heavy rain for mind-wandering with negative feedback: "the heavy rain was kind of negative, and in the heat it wasn't anything negative" (P1). Hence, the birdsong representing the state of being mindful were linked to positive feedback: "when I heard them [the birds] I just went 'yeah, I got it', and then I just immediately lost it [being mindful]" (P8).

Unfortunately, such easy recognition of meditation states with the aural feedback, fostered a judgmental attitude towards meditation session: "the water was nice but I think I was trying to like figure out the pattern [during the meditation], so maybe it was a bit too much" (P4). As a result, 7 participants have shown a more performance-oriented stance of their meditation, looking for the positive feedback, instead of experiencing the present moment in a non-judgmental manner, a key aspect of meditation training [13]: "it stressed me out when I had more rain, and when I didn't hear the birds I was like, oh I should be hearing the birds " (P7), "with the sound, I could hear the birds more when I was concentrated, so it kind of made me want to listen to the birds" (P3). As illustrated in these quotes, applying gamification principles such as rewards and punishments to meditation technologies may support awareness of meditation states but can be counterproductive as they hinder attention regulation. This is due to the fostered judgmental attitude rather than non-judgmental one, as an underlying principle of meditation [41].

In contrast to the judgmental stance emerging in aural condition, the warmth metaphors, with their ambiguous quality did not lead to it. In fact, the thermal patterns facilitated a non-judgmental attitude towards the experiences that arise during meditation: "I could kind of tell if I was losing focus [with the soundscape] and I felt I felt that pressure with it [during the meditation], [...] the heat was more gentle" (P4). As illustrated by this quote, the audio feedback was perceived as more disruptive hence added that layer of judgmental attitude towards being mindful, whereas the warmth was perceived as a gentler guidance in which they could choose whether or not pay attention to it: "It can help us [thermal feedback for meditation], I think, in some way without relying on it too much" (P1).

\section{DISCUSSION}

We now discuss the findings by revisiting the initial research starting by introducing the concept of interoceptive interaction, which we further extend through a discussion of the qualities of the embodied metaphors explored with the aural and thermal feedback.

\subsection{Interoceptive Interaction}

The exploration of the design space of meditation technologies through the Muse app and WarmMind prototype allowed the understanding of their distinct metaphors and qualities underpinning them. WarmMind is an illustration of a less explored class of technologies leveraging a specific form of interaction which we call interoceptive interaction. We define interoceptive interaction as interaction with bodily data through interoceptive senses aimed to bring the attention inwards to increase awareness of bodily states. This can have important wellbeing and health benefits, as interoceptive awareness is needed for regulation processes from emotional regulation to mindfulness meditation [23, 27, 29, 44, 67, 87].

Bodily data could include meditation states measured through brain activity, as well as emotional responses measured through skin conductance or heart rate, most of these explored in $\mathrm{HCl}$. The main distinction of this new class of technologies that we propose is that interaction with bodily data takes place through interoceptive 
senses rather than the predominantly used external senses via visual or aural interfaces (Figure 3 ) to communicate, for instance, embodied metaphors of meditation states [3, 64, 74, 91]. In contrast to the external senses which support interaction with the environment, interoceptive senses receive information from inside the body namely the viscera (i.e. respiratory, cardiovascular and gastrointestinal organs) and the skin (i.e. thermal and pain receptors) to support bodily optimal balance or homeostasis [67], as well as from the autonomous nervous system to support emotional awareness and regulation [52].

While modalities for interoceptive interaction have been less explored compared to traditional exteroceptive interaction, a rich starting point is haptics. Our exploration was focused on thermoception for attention regulation, while parallel $\mathrm{HCl}$ efforts have just started to emerge to communicate breathing through vibration patterns [54] or physiological arousal through heat [88] to support affect regulation. We suggest that interoceptive interaction can open a new design space for meditation technologies, in which the human body is not only used to monitor internal processes but is also used as a canvas to communicate meditation states via subtle actuations perceived to come from inside the body, and inspired by embodied metaphors such as our thermal patterns.

\subsection{Qualities of Embodied Metaphors for Meditation}

Our findings provide an overview of the embodied metaphors experienced during meditation, brought forward through two distinct mediums: an aural soundscape, and thermal patterns on the upper body. We now discuss the qualities of embodied metaphors and how these can support key aspects of meditation such as regulation of attention and attaining a non-judgmental stance. We also reflect on these qualities and their tensions in order to inform a design framework for meditation technologies.

\subsubsection{Qualities Impacting on Metaphor Understanding}

Participants' description of their experience of meditation with aural and thermal feedback emphasized that metaphors instantiated through the Muse's rainforest soundscape were easier to discover and understand than those provided by WarmMind. The mapped mindfulness and mind-wandering states as two opposite states on a continuum, one of which desirable, also indicates the polarity characteristic of the embodied schemata slowfast, which has been suggested as important for embodied tangible interaction [51]. However, the continuous aural feedback goes beyond polarity, by providing also the mapping for all the changes between these two key states. The change of state in Muse app maps transitions between the two polar states; albeit it does so in a rather symmetric manner, mapping in the same way any change of state. While easy to understand, hence discoverable, this symmetry is less supported by experiential accounts of expert meditators [19] according to whom the movement from mind-wandering to mindfulness is slow and gradual, but the reverse movement can be immediate. These insights suggest a more nuanced understanding of supporting discoverability, strongly advocated in tangible interaction research [51,69]. First, discoverability works best for the two polar states, but less so for the change of state. Our findings also indicate that one property of the sound stimuli that can vary consistently across the continuum between the polar states, is the one that drives the instantiation of image schemata (tempo for Muse), while the other sound properties can be leveraged to increase discoverability (timbre and pitch for Muse), and limit distraction (symmetric transition).

In contrast to the high discoverability of the embodied metaphors of the Muse app, the ones provided by the WarmMind prototype were less discoverable. Indeed, while all participants felt the warmth and most of them used it as a prompt to bring attention back to the body and breathing, the patterns were however not clearly 
associated with mindfulness and mind-wandering states. While challenging, ambiguity can also be a resource to design evocative systems intended to support more open interpretations [26, 71]. For instance, Sanches et al. argue for the use of ambiguity in the prototypes that rely on technologies that people have limited familiarity with, such as their prototype mapping skin conductance to arousal-based visualizations [71]. This resonates with our work, as we are targeting concepts that people are less familiar with such as thermal actuations, or are difficult to access and be aware of such as meditation states.

In the attempt to increase the rather limited expressiveness of the thermal patterns (for which we can only manipulate intensity and rhythm while our comparable, highly expressive Muse app manipulates four properties of sound), we decided to work with 4 actuators to more expressively communicate the random pattern for the mind-wandering state. On reflection, this was a less ideal choice, as it added more complexity than probably needed, thus challenging the understanding of this mapping. We can now see additional limitations with the movement metaphor, as we start asking ourselves what is the movement about? What is meant to be moved? To answer these questions, we reflected back on cognitive linguistic findings where the movement is described as one of a preoccupied mind, the movement of chaotic thoughts [42, 79]. For WarmMind, however, the warmthbased embodied movement would do better to reflect the body rather than abstract movement of thoughts, as the random pattern does little to support the bodily awareness during mind-wandering moments. In fact, it does the opposite, by bringing attention to something less structured, it can hinder focus of attention when is most needed to be regained.

\subsubsection{Qualities Impacting on Attention Regulation}

Findings indicate that while Muse app supported better discoverability of the metaphorical mappings, it was also more distracting as participants tried to regulate their attention. In contrast, while less discoverable, WarmMind appears to be better suited to support regulation of attention. Several qualities of embodied metaphors appear to have contributed to this outcome, the most important being interoceptive and subtleness of the WarmMind vs exteroceptive and richness of the Muse app.

Findings indicate that with its inwards focus on the body, the WarmMind prototype remained in the background. Due to its low intensity actuation (warmth rather than hot or cold), sampled discretely based on changes of meditation states rather than continuously, and its lower expressiveness, conveyed through the fewer properties of thermal actuators compared to sound ones; WarmMind allowed for the main focus of attention to remain on the breathing, rather than being required to continuously attend to the interaction itself. These outcomes contribute to the $\mathrm{HCl}$ work on subtle interaction [63] and extend the previous emphasis on multimodality [35] in tangible interaction, towards interoceptive modality and particularly thermoception. They also highlight the challenge of continuous feedback, as regulating attention is a key skill that meditation practice aims to support, albeit not trivial to develop [90]. During meditation, it is easy to engage with distracting thoughts or external stimuli, hence novice meditators usually rely on support to help them bring attention back to the present moment e.g. audio instructions [20], prescribed movements [76] or interactive technologies [80, 84]. Whilst in traditional meditation such support is provided in a discrete manner e.g. the teacher says a generic statement every few minutes to foster bringing the attention back to the present moment [41], most interactive technologies provide a continuous stream of feedback adapting in real-time to the changes in meditation states (Figure 3). Our outcomes indicate that for FAM, a more discrete type of feedback may be more beneficial. 
An interesting outcome is that, although not significant, MAAS questionnaire has shown larger increase in mindfulness state after using the Muse app. This is surprising given participants' increased challenges for attention regulation experienced while using Muse app. It may be that our two interfaces supported mindfulness state through different mechanisms, and future work is needed to explore this. It is worth highlighting that Muse app's nature-based soundscape was characterized by white noise consisting of different sounds at multiple frequencies with limited changes in rhythm or pitch [92], similar to other sounds encountered in nature, created for instance by rivers, ocean waves, wind blowing through vegetation, rain or fire. White noise is frequently used in music therapy and consistent findings have shown its impact on increased positive emotions, and relaxation [21, 82]. Moreover, consistent findings have also shown the link between mindfulness and connectedness with nature, as well nature's restorative value for our attention capability [77], allowing for "relatively effortless attention and processing in an environment to which humans are evolutionarily adapted" [28] [p. 10].

\subsubsection{Qualities Supporting Non-judgmental Stance}

Findings indicate that unlike WarmMind prototype, Muse app has led most participants to take a judgmental stance regarding their meditation session. Several qualities facilitated this, such as high discoverability [51, 69], rich and continuous feedback allowing participants to know moment by moment how well or less well they were doing, and in particular polarity coupled with gamification principle [80]. According to this, birdsongs, mapped to mindfulness state, were particularly distinct (different timbre) and easy to perceive as reward, whereas heavy rain mapped to mind-wandering state was perceived as negative feedback. In turn, this led to a judgmental stance towards meditation states, as participants felt compelled to maintain the mindfulness state to keep hearing the songbirds, and to transition out from mind-wandering to no longer hear the heavy rain. In other words, they started experiencing attachment towards the meditation's goal rather than being present within the meditation process. This contrasts a key tenant of meditation practice, namely the non-judgmental acceptance of all experiences that may arise during it [90].

\subsection{IMPLICATIONS FOR DESIGN}

We now report on the value of our findings for the design of meditation technologies highlighting the need for deep understanding of the metaphors to design for, the tension of supporting both discoverability and attention regulation, and for sensitive design of the mapping for being mindful.

\subsubsection{Understanding and Experiencing the Embodied Metaphors}

Our findings indicate the importance of designers' deep understanding of the metaphor, its origin and what it can be applied to, without altering aspects which would make it less effective, i.e. in our case the chaotic movement of thoughts to the random movement of warmth on the body. We argue for the benefit of drawing from multiple sources to understand the metaphors [35] such as spiritual tradition, cognitive linguistics, and phenomenology of meditation, engaging in first person somadesign exploration of the metaphors, and running user studies with contrasting prototypes to better understand how the metaphors work or not. We also call for $\mathrm{HCl}$ research to articulate the rationale of the chosen metaphors and of image schemata instantiating them, so can share and build resources for designing with metaphors in general such as tangible interaction, or technologies for affect regulation [54,89], and for attention regulation in particular. 


\subsubsection{Supporting Discoverability of Embodied Metaphors}

Our findings indicate that many qualities of embodied metaphors support their understanding. The following list, illustrated with examples from Muse app and WarmMind, offers a valuable resource to consider when designing for discoverability: (i) type of interaction with exteroceptive being easier to understand than exteroceptive; (ii) increased stimulation and expressiveness as higher number of modality properties being manipulated for the design of the metaphors: 4 vs 2, timbre, tempo, volume and pitch vs rhythm and intensity, (iii) pace at which metaphors are presented: quick and continuous for representing major changes from one meditation state to another as well as minor ones vs slow and discrete triggered only by major changes from one meditation state to another; (iv) high polarity: two main states; (v) ambiguity: low vs high; (vi) gamification: reward for mindfulness vs punitive for mindlessness; (vi) nature-inspired content.

\subsubsection{Supporting Attention Regulation and Non-Judgmental Stance}

Findings also indicate qualities that particularly support attention regulation and non-judgmental stance: (i) type of interaction: interoceptive rather than exteroceptive, (ii) limited stimulation and expressiveness as lower number of modalities and properties being manipulated, (iii) pace of metaphors' presentation: slow and discrete vs quick and continuous, (iv) moderate polarity: two states; (v) ambiguity: high vs low; (vi) no gamification.

\subsubsection{Sensitive Mapping for Mindlessness State}

Findings indicate the mindlessness with its lack of awareness of bodily states is not easy to design for. Moreover, any intense stimulation design to attract attention may be perceived as punitive. Thus, we argue for the value of sensitively mapping the problematic mindlessness state not so much by mirroring it, but to better support its attention regulation. To a less extent, a similar approach has been seen in Muse app through its symmetric transition representing the movement between the two polar states. We will go even further to suggest metaphors for mind-wandering state that only do not attract attention, but which purposefully support its regulation (see below).

\subsubsection{Thermal-based Metaphors for Meditation}

For future thermal-based feedback for meditation technologies, we suggest exploring the warmth metaphor as dominant (rather than movement metaphor), mapping the higher, radiant warmth to mindfulness state, and less warmth to mind-wandering state (rather than cold as we considered in our initial design exploration). The image schemata will be high-low, emphasizing both high-low warmth intensities, and high-low placement of thermal actuators on the body: chest for mindfulness, belly for mind-wandering. Thus, the metaphor of mind-wandering state will be warmth aimed not to emphasize awareness of mind wandering, but for actually help the meditator bring their attention to the breathing in the belly. We also suggest working with the low expressiveness of the thermal properties without attempting to heighten it at the expense of discoverability, for instance using no more than 2 actuators, enough to communicate the two main states. 

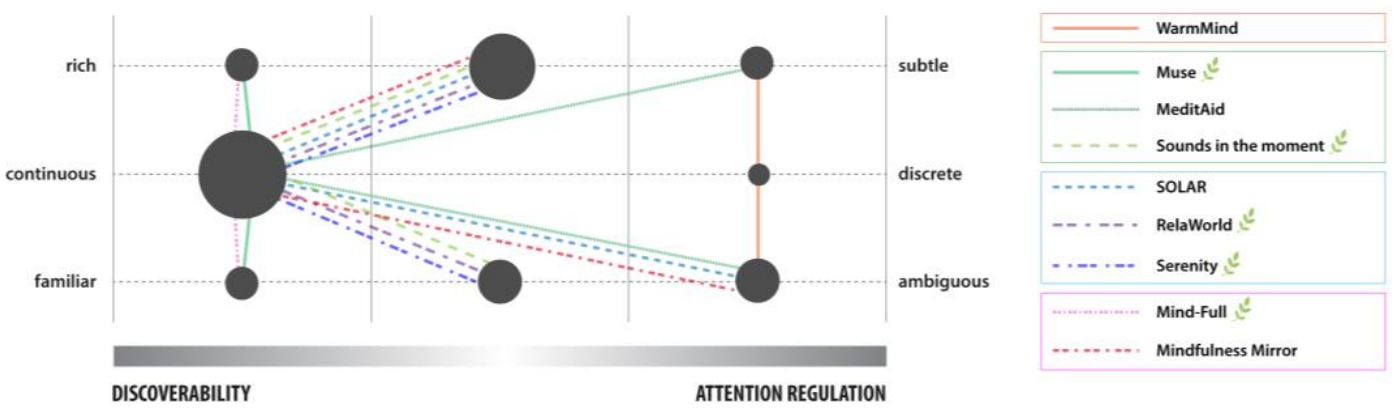

Figure 3. Three qualities of embodied metaphors mapping meditation states: sensory stimulation (rich vs subtle), temporal aspects (continuous vs discrete feedback), and support for understanding (familiar vs ambiguous mappings). Furthermore, a color scheme represents the interface modality: green for auditory interfaces (i.e. Muse and [18, 74]), blue for VR environments with audiovisual feedback (i.e. [16, 45, 65]), pink for visual displays (i.e. [6, 36]), and orange for interoceptive interface (i.e. WarmMind). Finally, designs that use representational nature-based metaphors are marked with a leaf $\$$, whilst the other use abstract metaphors.

\subsection{Framework for Designing Meditation Technologies}

To better support a metaphor inspired approach to designing for meditation, we describe an initial framework capturing the main qualities of the embodied metaphors that our findings highlighted, and the relative tension between them. This is intended to summarize in a concise manner the richness of our data. We also describe its evaluation, as we have used it to populate it not only the two systems we have explored: Muse app and WarmMind prototype, but also with 7 additional meditation technologies we reviewed in the state-of-the-art which employed neurofeedback-based embodied metaphors on exteroceptive modalities such as visual, auditory interfaces and VR environment. The framework is organized to reflect the tension highlighted by our findings between discoverability on the one hand, and attention regulation on the other hand. Then, this tension was further broken down in 3 of the key qualities supporting them both, albeit in opposing ways: sensory stimulation (rich or subtle), temporal aspects (continuous or discrete feedback) and ambiguity (familiar or ambiguous mapping). Given the importance of nature-inspired design in Muse app, we provided another layer to the framework by marking with the leaf the systems employing nature-inspired metaphors.

Figure 3 shows the framework populated with all 9 systems, each one across each of the three horizontal qualities, with the size of the circle representing the number of systems in that space. For instance, Muse is rich in sensory stimulation (top left), provides continuous feedback (middle left), and has a familiar mapping (bottom left). As illustrated, most neurofeedback meditation systems in $\mathrm{HCl}$ provide a moderate level of sensory stimulation, continuous feedback and moderate to ambiguous mappings. For example, in Relaworld the aesthetics of the virtual scene are intentionally minimal to modestly capture attention without eliciting mental fatigue and cognitive overload, i.e. only display the ocean and the sky in a range of gray-scale shades, yet supporting discoverability by being continuous and nature-based mapping [45]. Nevertheless, the exploration of the qualities of subtle and discrete feedback have been less explored in current interactive systems for meditation, yet our findings with WarmMind suggest their benefit to the practices of interoception and attention regulation during meditation.

Also, whilst traditional guided meditation practices account for this by providing temporal-based discrete feedback, all the neurofeedback systems for meditation in $\mathrm{HCl}$ provide a continuous feedback mapping all 
changes in meditation states in real-time. Although this highly support discoverability, we found it may also overwhelm the meditator and challenge the regulation of attention. WarmMind provides an event-based discrete feedback, i.e. tailored to the meditator's experience but requiring a lower engagement with the technology hence facilitating attention regulation, which is a space that has not been explored for meditation technologies and our findings support their potential benefits for meditation.

\section{CONCLUSIONS}

Meditation is a mind-body practice with considerable wellbeing benefits which can take different forms. Most meditation technologies employ metaphorical mappings of meditation states to visual or soundscape representations to support awareness of mind-wandering and attention regulation, although the rationale for such mappings is seldom articulated. Moreover, such external modalities also take the focus attention away from the body. We advance the concept of interoceptive interaction and employed embodied metaphor theory to explore the design of mappings to the interoceptive sense of thermoception. We illustrate this concept with WarmMind, an on-body interface integrating heat actuators for mapping meditation states. We report on an exploratory study with 10 participants comparing our novel thermal metaphors for mapping meditation states with comparable ones, albeit in aural modality, as provided by Muse meditation app. To our understanding, this is one of the few instances of a cross-modal comparison of meditation technologies. Our findings indicate that whilst the soundscape was perceived as an external and expressive feedback modality, thermal patterns were felt as subtle experiences coming from inside the body. We discuss the qualities of embodied metaphors and advance a framework for mapping bodily experiences onto interface modality and content.

\section{ACKNOWLEDGMENTS}

\section{REFERENCES}

[1] Alfaras, M. et al. 2020. From Biodata to Somadata. Proceedings Computer in Human Factors CHI 2020. ACM. (2020).

[2] El Ali, A. et al. ThermalWear: Exploring Wearable On-chest Thermal Displays to Augment Voice Messages with Affect. DOI:https://doi.org/10.1145/3313831.3376682.

[3] Amores, J. et al. 2016. PsychicVR: Increasing mindfulness by using virtual reality and brain computer interfaces. Proceedings of the 2016 CHI Conference Extended Abstracts on Human Factors in Computing Systems - CHI EA '16 (New York, New York, USA, 2016), 2-2.

[4] Antle, A.N. et al. 2009. Designing to support reasoned imagination through embodied metaphor. C and C 2009-Proceedings of the 2009 ACM SIGCHI Conference on Creativity and Cognition (2009).

[5] Antle, A.N. et al. 2019. Evaluating the impact of a mobile neurofeedback app for young children at school and home. Conference on Human Factors in Computing Systems - Proceedings (May 2019).

[6] Antle, A.N. et al. 2018. Opening up the design space of neurofeedback brain-computer interfaces for children. ACM Transactions on Computer-Human Interaction. 24, 6 (Jan. 2018). DOI:https://doi.org/10.1145/3131607.

[7] Antle, A.N. et al. 2008. Playing with the sound maker: do embodied metaphors help children learn? Proceedings of the 7th International Conference on Interaction Design and Children, IDC 2008 (2008).

[8] Antle, A.N. et al. 2009. What the body knows: Exploring the benefits of embodied metaphors in hybrid physical digital environments. Interacting with Computers. 21, 1-2 (Jan. 2009), 66-75. DOI:https://doi.org/10.1016/j.intcom.2008.10.005.

[9] Bakkers, M. et al. 2013. Temperature threshold testing: a systematic review. Journal of the peripheral nervous system : JPNS.

[10] Basnett, D. 2016. LOOKING INWARDS, SPEAKING OUT: EXPLORING MEDITATION WITH NOVICE MEDITATORS TAKING PART IN A SHORT-TERM MEDITATION PROGRAM.

[11] Blackwell, A.F. 2006. The reification of metaphor as a design tool. ACM Transactions on Computer-Human Interaction.

[12] Bødker, S. 2006. When second wave $\mathrm{HCl}$ meets third wave challenges. Proceedings of the 4th Nordic conference on Human- 
computer interaction changing roles - NordiCHI '06 (New York, New York, USA, 2006), 1-8.

[13] Brown, K.W. and Ryan, R.M. 2003. The benefits of being present: Mindfulness and its role in psychological well-being. Journal of Personality and Social Psychology. 84, 4 (2003), 822-848. DOl:https://doi.org/10.1037/0022-3514.84.4.822.

[14] Bumatay, A.L. and Seo, J.H. 2015. Investigating the role of haptic stimulation in mobile meditation tools. Communications in Computer and Information Science (2015), 451-456.

[15] Carroll, J.M. et al. 1988. Interface Metaphors and User Interface Design. Handbook of Human-Computer Interaction.

[16] Choo, A. and May, A. 2015. Virtual mindfulness meditation: Virtual reality and electroencephalography for health gamification. Conference Proceedings - 2014 IEEE Games, Media, Entertainment Conference, IEEE GEM 2014 (Feb. 2015).

[17] Claus, D. et al. 1987. Methods of measurement of thermal thresholds. Acta Neurologica Scandinavica. (1987) DOI:https://doi.org/10.1111/j.1600-0404.1987.tb03583.x.

[18] Cochrane, K.A. et al. 2018. Sounds in the moment: Designing an Interactive EEG Nature Soundscape for Novice Mindfulness Meditators. Proceedings of the 30th Australian Conference on Computer-Human Interaction - OzCHI '18 (2018), 298-302.

[19] Daudén Roquet, C. and Sas, C. 2020. Body Matters: Exploration of the Human Body as a Resource for the Design of Technologies for Meditation. Proceedings of Designing Interactive Systems, DIS 2020, ACM. May (2020). DOI:https://doi.org/10.1145/3357236.3395499.

[20] Daudén Roquet, C. and Sas, C. 2018. Evaluating Mindfulness Meditation Apps. Extended Abstracts of the 2018 CHI Conference on Human Factors in Computing Systems (2018), 1-6.

[21] DeLoach, A.G. et al. 2015. Tuning the cognitive environment: Sound masking with "natural" sounds in open-plan offices. The Journal of the Acoustical Society of America. (2015). DOI:https://doi.org/10.1121/1.4920363.

[22] Esch, T. 2014. The Neurobiology of Meditation and Mindfulness.

[23] Farb, N. et al. 2015. Interoception, contemplative practice, and health. Frontiers in Psychology. 6, (Jun. 2015$), 763$. DOI:https://doi.org/10.3389/fpsyg.2015.00763.

[24] Fereday, J. and Muir-Cochrane, E. 2006. Demonstrating Rigor Using Thematic Analysis: A Hybrid Approach of Inductive and Deductive Coding and Theme Development. International Journal of Qualitative Methods. 5, 1 (Mar. 2006), 80-92. DOI:https://doi.org/10.1177/160940690600500107.

[25] Fujino, M. et al. 2018. Open monitoring meditation reduces the involvement of brain regions related to memory function. Scientific Reports. (2018). DOI:https://doi.org/10.1038/s41598-018-28274-4.

[26] Gaver, W.W. et al. 2003. Ambiguity as a resource for design. Proceedings of the conference on Human factors in computing systems - CHI '03 (New York, New York, USA, 2003), 233.

[27] Gibson, J. 2019. Mindfulness, Interoception, and the Body: A Contemporary Perspective. Frontiers in Psychology. (2019). DOI:https://doi.org/10.3389/fpsyg.2019.02012.

[28] Hamann, G.A. and Ivtzan, I. 2016. Social Inquiry into Well-Being 30 Minutes in Nature a Day Can Increase Mood, Well-Being, Meaning in Life and Mindfulness: Effects of a Pilot Programme. Social Inquiry into Well-Being. 2, 2 (Oct. 2016$), 34-46$. DOI:https://doi.org/10.13165/SIIW-16-2-2-04.

[29] Hanley, A.W. et al. 2017. Holding the body in mind: Interoceptive awareness, dispositional mindfulness and psychological wellbeing. Journal of Psychosomatic Research. 99, (Aug. 2017), 13-20. DOI:https://doi.org/10.1016/J.JPSYCHORES.2017.05.014.

[30] Hasenkamp, W. et al. 2012. Mind wandering and attention during focused meditation: A fine-grained temporal analysis of fluctuating cognitive states. Neurolmage. 59, 1 (Jan. 2012), 750-760. DOI:https://doi.org/10.1016/J.NEUROIMAGE.2011.07.008.

[31] Höök, K. 2009. Affective loop experiences: designing for interactional embodiment. Philosophical Transactions of the Royal Society B: Biological Sciences. (2009). DOl:https://doi.org/10.1098/rstb.2009.0202.

[32] Höök, K. et al. 2018. Embracing first-person perspectives in soma-based design. Informatics. MDPI Multidisciplinary Digital Publishing Institute.

[33] Höök, K. et al. 2016. Somaesthetic Appreciation Design. Proceedings of the 2016 CHI Conference on Human Factors in Computing Systems - CHI '16 (New York, New York, USA, 2016), 3131-3142.

[34] Hornecker, E. 2012. Beyond affordance: Tangibles' hybrid nature. Proceedings of the 6th International Conference on Tangible, Embedded and Embodied Interaction, TEI 2012 (2012).

[35] Hurtienne, J. 2017. How Cognitive Linguistics Inspires HCl: Image Schemas and Image-Schematic Metaphors. International Journal of Human-Computer Interaction. (2017). DOI:https://doi.org/10.1080/10447318.2016.1232227.

[36] James-Reynolds, C. and Currie, E. 2019. Mindfulness Mirror. Lecture Notes in Computer Science (including subseries Lecture Notes in Artificial Intelligence and Lecture Notes in Bioinformatics) (Dec. 2019), 456-461.

[37] Johnson, M. 1989. The Body in the Mind: The Bodily Basis of Meaning, Imagination, and Reason. The Journal of Aesthetics and Art Criticism. (1989). DOl:https://doi.org/10.2307/431155.

[38] Jones, L.A. and Berris, M. 2002. The psychophysics of temperature perception and thermal-interface design. Proceedings - 10th Symposium on Haptic Interfaces for Virtual Environment and Teleoperator Systems, HAPTICS 2002 (2002).

[39] Jones, L.A. and Ho, H.N. 2008. Warm or cool, large or small? The challenge of thermal displays. IEEE Transactions on Haptics. (2008). DOI:https://doi.org/10.1109/TOH.2008.2.

[40] Jonsson, M. et al. 2016. The aesthetics of heat: Guiding awareness with thermal stimuli. TEl 2016 - Proceedings of the 10th Anniversary Conference on Tangible Embedded and Embodied Interaction (2016).

[41] Kabat-Zinn, J. 2009. Wherever you go, there you are: mindfulness meditation in everyday life. Hyperion e-book. 
[42] Kang, B. 2018. Unifying opposites through metaphor: A cognitive approach to the Buddhist metaphors for the mind in the Awakening of Faith discourse. Religions. (2018). DOl:https://doi.org/10.3390/rel9110345.

[43] Kerr, C.E. et al. 2013. Mindfulness starts with the body: somatosensory attention and top-down modulation of cortical alpha rhythms in mindfulness meditation. Frontiers in Human Neuroscience. 7, (2013), 12. DOl:https://doi.org/10.3389/fnhum.2013.00012.

[44] Kok, B.E. and Singer, T. 2017. Phenomenological Fingerprints of Four Meditations: Differential State Changes in Affect, MindWandering, Meta-Cognition, and Interoception Before and After Daily Practice Across 9 Months of Training. Mindfulness. (2017). DOI:https://doi.org/10.1007/s12671-016-0594-9.

[45] Kosunen, I. et al. 2016. RelaWorld: Neuroadaptive and Immersive Virtual Reality Meditation System. Iui 2016. (2016), 208-217. DOI:https://doi.org/10.1145/2856767.2856796.

[46] Krigolson, O.E. et al. 2017. Choosing MUSE: Validation of a low-cost, portable EEG system for ERP research. Frontiers in Neuroscience. 11, MAR (2017), 109. DOI:https://doi.org/10.3389/fnins.2017.00109.

[47] Lakoff, G. and Johnson, M. 2013. Metaphors We Live By.

[48] Langacker, R.W. and Lakoff, G. 1988. Women, Fire, and Dangerous Things: What Categories Reveal about the Mind. Language. (1988). DOl:https://doi.org/10.2307/415440.

[49] Langston, W. 2002. Violating Orientational Metaphors Slows Reading. Discourse Processes. (2002). DOI:https://doi.org/10.1207/s15326950dp3403_3.

[50] Loke, L. and Schiphorst, T. 2018. The somatic turn in human-computer interaction. Interactions. (2018).

[51] Macaranas, A. et al. 2015. What is Intuitive Interaction? Balancing Users' Performance and Satisfaction with Natural User Interfaces. Interacting with Computers. (2015). DOI:https://doi.org/10.1093/iwc/iwv003.

[52] Mehling, W. 2016. Differentiating attention styles and regulatory aspects of self-reported interoceptive sensibility. Philosophical Transactions of the Royal Society B: Biological Sciences.

[53] Mehling, W.E. et al. 2012. The Multidimensional Assessment of Interoceptive Awareness (MAIA). PLoS ONE. (2012). DOI:https://doi.org/10.1371/journal.pone.0048230.

[54] Miri, P. et al. 2020. Evaluating a Personalizable, Inconspicuous Vibrotactile(PIV) Breathing Pacer for In-the-Moment Affect Regulation. Proceedings of the 2020 CHI Conference on Human Factors in Computing Systems (2020), 1-12.

[55] Mohamed, M. et al. 2017. Understanding the Role of Human Senses in Interactive Meditation. Proceedings of the 2017 CHI Conference on Human Factors in Computing Systems - CHI '17 (New York, New York, USA, 2017), 4960-4965.

[56] Moran, S. et al. ExoPranayama: a biofeedback-driven actuated environment for supporting yoga breathing practices. Personal and Ubiquitous Computing. 20. DOI:https://doi.org/10.1007/s00779-016-0910-3.

[57] Museтм - Meditation Made Easy with the Muse Headband: https://choosemuse.com/. Accessed: 2019-07-07.

[58] Nash, J.D. and Newberg, A. 2013. Toward a unifying taxonomy and definition for meditation. Frontiers in psychology. 4, (2013), 806. DOI:https://doi.org/10.3389/fpsyg.2013.00806

[59] Patibanda, R. et al. 2017. Life Tree: Understanding the Design of Breathing Exercise Games. Proceedings of the Annual Symposium on Computer-Human Interaction in Play - CHI PLAY'17 (New York, New York, USA, 2017), $19-31$.

[60] Perlman, D.M. et al. 2010. Differential Effects on Pain Intensity and Unpleasantness of Two Meditation Practices. Emotion. (2010). DOI:https://doi.org/10.1037/a0018440.

[61] Petitmengin, C. et al. 2019. Studying the experience of meditation through Micro-phenomenology. Current Opinion in Psychology. Elsevier B.V.

[62] Pietrzak, T. and Wanderley, M.M. 2020. Haptic and audio interaction design. Journal on Multimodal User Interfaces.

[63] Pohl, H. et al. 2019. Charting subtle interaction in the $\mathrm{HCl}$ literature. Conference on Human Factors in Computing Systems Proceedings (2019).

[64] Prpa, M. et al. 2018. Attending to Breath: Exploring How the Cues in a Virtual Environment Guide the Attention to Breath and Shape the Quality of Experience to Support Mindfulness. Proceedings of the 2018 on Designing Interactive Systems Conference 2018 - DIS '18 (New York, New York, USA, 2018), 71-84.

[65] Prpa, M. et al. 2016. Hacking alternatives in 21stcentury: Designing a bio-responsive virtual environment for stress reduction. Communications in Computer and Information Science (Sep. 2016), 34-39.

[66] Richer, R. et al. 2018. Real-time Mental State Recognition using a Wearable EEG. 2018 40th Annual International Conference of the IEEE Engineering in Medicine and Biology Society (EMBC) (Jul. 2018), 5495-5498.

[67] Ritchie, J.B. and Carruthers, P. 2015. Oxford Handbooks Online The Bodily Senses 1 The Interoceptive Senses. November 2018 (Jul. 2015), 1-22. DOI:https://doi.org/10.1093/oxfordhb/9780199600472.013.026.

[68] Roney-Dougal, S.M. and Solfvin, J. 2011. Exploring the relationship between tibetan meditation attainment and precognition. Journal of Scientific Exploration. (2011).

[69] Roo, J.S. et al. 2017. Inner Garden: Connecting Inner States to a Mixed Reality Sandbox for Mindfulness. Proceedings of the 2017 CHI Conference on Human Factors in Computing Systems - CHI '17 (New York, New York, USA, 2017), 1459-1470.

[70] Saffer, D. 2005. The Role of Metaphor in Interaction Design. Citeseer. (2005). DOI:https://doi.org/10.1207/s15327868ms2003_1.

[71] Salehzadeh Niksirat, K. et al. 2017. A Framework for Interactive Mindfulness Meditation Using Attention-Regulation Process. Proceedings of the 2017 CHI Conference on Human Factors in Computing Systems - CHI '17 (New York, New York, USA, 2017), $2672-2684$. 
[72] Sanches, P. et al. 2019. Ambiguity as a resource to inform proto-practices: The case of skin conductance. ACM Transactions on Computer-Human Interaction (TOCHI). 10, 20 (2019).

[73] Sanches, P. et al. 2010. Mind the body!: designing a mobile stress management application encouraging personal reflection. Proceedings of the 8th ACM Conference on Designing Interactive Systems - DIS '10 (New York, New York, USA, 2010), 47-56.

[74] Sas, C. et al. 2014. Generating implications for design through design research. Proceedings of the 32nd annual ACM conference on Human factors in computing systems - CHI '14. (2014), 1971-1980. DOI:https://doi.org/10.1145/2556288.2557357.

[75] Sas, C. and Chopra, R. 2015. MeditAid: a wearable adaptive neurofeedback-based system for training mindfulness state. Personal and Ubiquitous Computing. 19, 7 (Oct. 2015), 1169-1182. DOI:https://doi.org/10.1007/s00779-015-0870-z.

[76] Scherer, B. and Waistell, J. 2018. METAPHORS OF MINDFULNESS. Contemporary Buddhism. (2018). DOI:https://doi.org/10.1080/14639947.2018.1479557.

[77] Schmalzl, L. et al. 2014. Movement-based embodied contemplative practices: definitions and paradigms. Frontiers in Human Neuroscience. 8, (2014), 205. DOI:https://doi.org/10.3389/fnhum.2014.00205.

[78] Schutte, N.S. and Malouff, J.M. 2018. Mindfulness and connectedness to nature: A meta-analytic investigation. Personality and Individual Differences. (2018). DOI:https://doi.org/10.1016/j.paid.2018.01.034.

[79] Shusterman, R. 2008. Body Consciousness: A Philosophy of Mindfulness and Somaesthetics.

[80] Silvestre-López, A.J. and Ferrando, I.N. i 2017. Metaphors in the conceptualisation of meditative practices. Metaphor and the Social World. (2017). DOI:https://doi.org/10.1075/msw.7.1.03sil.

[81] Sliwinski, J. et al. 2017. A Review of Interactive Technologies as Support Tools for the Cultivation of Mindfulness. Mindfulness Springer US.

[82] Snyder, J. et al. 2015. MoodLight. Proceedings of the 18th ACM Conference on Computer Supported Cooperative Work \& Social Computing - CSCW'15 (New York, New York, USA, 2015), 143-153.

[83] Stanchina, M.L. et al. 2005. The influence of white noise on sleep in subjects exposed to ICU noise. Sleep Medicine. (2005). DOI:https://doi.org/10.1016/j.sleep.2004.12.004.

[84] Strang, C. 1961. XIV-The Perception of Heat. Proceedings of the Aristotelian Society. (1961). DOI:https://doi.org/10.1093/aristotelian/61.1.239.

[85] Terzimehić, N. et al. 2019. A review \& Analysis of mindfulness research in $\mathrm{HCl}$ framing current lines of research and future opportunities. Conference on Human Factors in Computing Systems - Proceedings (New York, New York, USA, 2019), 1-13.

[86] Thaisetthawatkul, P. 2014. Quantitative sensory testing. Neuromuscular Disorders in Clinical Practice.

[87] The Mindful Attention Awareness Scale (MAAS) - State: https://ggsc.berkeley.edu/images/uploads/The_Mindful_Attention_Awareness_Scale__State.pdf. Accessed: 2020-09-04.

[88] Treves, I.N. et al. 2019. The relationship between mindfulness and objective measures of body awareness: A meta-analysis. Scientific Reports. (2019). DOI:https://doi.org/10.1038/s41598-019-53978-6.

[89] Umair, M. et al. 2018. Dynamic Displays at Wrist for Real Time Visualization of Affective Data. Proceedings of the 19th International ACM SIGACCESS Conference on Computers and Accessibility - DIS '18 (New York, New York, USA, 2018), 201205.

[90] Umair, M. et al. 2019. Towards affective chronometry: Exploring smart materials and actuators for real-time representation of changes in arousal. Proceedings of the 2019 on Designing Interactive Systems Conference (2019), 1479-1494.

[91] Vago, D.R. and Silbersweig, D.A. 2012. Self-awareness, self-regulation, and self-transcendence (S-ART): a framework for understanding the neurobiological mechanisms of mindfulness. Frontiers in Human Neuroscience. 6, (Oct. 2012), 296. DOI:https://doi.org/10.3389/fnhum.2012.00296

[92] Vidyarthi, J. et al. 2012. Sonic Cradle. Proceedings of the Designing Interactive Systems Conference (New York, New York, USA 2012), 408

[93] Yu, B. et al. 2018. Unwind: a musical biofeedback for relaxation assistance. Behaviour and Information Technology. (2018). DOI:https://doi.org/10.1080/0144929X.2018.1484515. 